Research Paper

\title{
Mesenchymal Stem Cells Accelerate the Remodeling of Bladder VX2 Tumor Interstitial Microenvironment by TGF 1 1-Smad Pathway
}

\author{
Qingya Yang1, Jun Chen ${ }^{1,2}$, Yaofeng Zhu' ${ }^{2}$, Zhishun $\mathrm{Xu}^{2}$ \\ 1. Department of Urology, Qilu Hospital (Qingdao), Shandong University, Qingdao 266035, China. \\ 2. Department of Urology, Qilu Hospital, Shandong University, Jinan 250012, China \\ $\triangle$ Corresponding author: Jun Chen, Department of Urology, Qilu Hospital, Shandong University, 107 Wenhuaxi Road, Jinan 250012, China; \\ chenjunxinxiang@163.com
}

(1) The author(s). This is an open access article distributed under the terms of the Creative Commons Attribution License (https://creativecommons.org/licenses/by/4.0/). See http://ivyspring.com/terms for full terms and conditions.

Received: 2018.10.18; Accepted: 2019.04.23; Published: 2019.07.25

\begin{abstract}
Background: Mesenchymal stem cells (MSCs) have been proved to be able to differentiate into cells that are conducive to tumor growth and invasion. The mechanism is not clear. This present study was aimed to find out whether TGF $\beta 1$-Smad pathway was involved in this process.

Methods: For the in vitro experiment, five groups of MSCs were cultured to test whether VX2 culture supernatant could induce the differentiation of MSCs into myofibroblasts. And then transforming growth factor $\beta 1$ (TGF $\beta 1$ ) receptor or Smad2 of MSCs were blocked by RNA interference technique to test whether TGF $\beta 1$-Smad pathway was involved in the differentiation. In the animal experiment, different kinds of MSCs were co-inoculated with VX2 cells in bladder to test whether the blockage of TGF $\beta 1$ receptor or Smad2 of MSCs could affect the expression of TGF 31 , epidermal growth factor (EGF), fibroblast activation protein alpha (FAPa), and matrix metalloprotein 9 (MMP9) in five animal groups.

Results: VX2 culture supernatant could up-regulate the expression of $\alpha$-SMA and Vimentin in MSCs, which indicated that VX2 culture supernatant could induce the differentiation of MSCs into myofibroblasts. Either the Blockage of TGF $\beta 1$ receptor or Smad2 of MSCs could lead to decreased expression of $\alpha$-SMA and Vimentin in MSCs. In the animal experiment, MSCs could favor VX2 bladder tumor growth and up-regulate the expression of TGFB1, EGF, FAPa, MMP9 in VX2 tumor tissue. However, when TGF $\beta 1$ receptor or Smad2 of MSCs was blocked, the above effects were attenuated.

Conclusions: Under the induction of tumor microenvironment, MSCs can differentiate into myofibroblasts and then affect tumor interstitial microenvironment remodeling. This process is mediated by TGF $\beta 1$-Smad2 pathway.
\end{abstract}

Key words: Mesenchymal stem cells; tumor; stroma remodeling

\section{Introduction}

Mesenchymal stem cells (MSCs) are widely distributed in the body, mainly in the bone marrow. They have been widely used in tissue engineering, cells and gene therapy. Regarding to how the target organ induces MSCs' migration, it is generally thought that the extracellular signals emitted by the injured organ regulate this process, and various chemokine receptors expressed by MSCs are closely

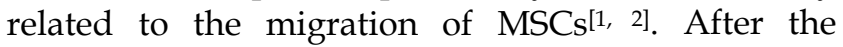
migration of MSCs to target organs, their further differentiation is environmental-dependent ${ }^{[3,4]}$.

As a rapidly growing and metabolically active disease, tumor can also induce MSCs to differentiate into cells that are conducive to tumor growth and invasion. Current research results have indeed confirmed this hypothesis ${ }^{[5]}$. Studeny et al transferred adenovirus vectors carrying the $\beta$-interferon gene into MSCs and found a large number of MSCs in tumor tissue[6]. These experimental results show that tumor cells do have the ability to induce the migration of 
MSCs. Other related studies also found similar results ${ }^{[7,8]}$. Furthermore, studies also found that MSCs could promote tumor growth and metastasis ${ }^{[9-11]}$.

Our previous research results showed that under the induction of tumor microenvironment, MSCs could differentiate into myofibroblasts, and further accelerate the development of tumors by promoting tumor interstitial microenvironment remodeling $[10,12]$. The mechanism of tumor-induced differentiation of MSCs into myofibroblasts remains unclear. In the study of lysophosphatidic acid-induced differentiation of MSCs into myofibroblasts, Jeon et al found that blocking the expression of Smad2/3 would significantly reduce the expression of a-SMA, while blocking the binding of transforming growth factor $\beta 1$ (TGF $\beta 1$ ) to its receptor could reduce the aSMA expression and Smad2 phosphorylation [13]. This result suggests that TGF $\beta 1$-Smad signaling pathway plays an important role in the differentiation of MSCs into myofibroblasts. In view of the important role of myofibroblasts in the remodeling of tumor interstitial microenvironment, the blockage of TGF $\beta 1-S m a d$ signaling pathway in MSCs may simultaneously affect the remodeling of tumor interstitial microenvironment and further affect the development of tumor. This study mainly validated the above hypothesis. And the remodeling of tumor interstitial microenvironment was shown by the expression of TGF $\beta 1$, epidermal growth factor (EGF), fibroblast activation protein alpha (FAPa), matrix metalloprotein 9 (MMP9).

\section{Materials and Methods}

\section{The design of the study}

MSCs have been proved to be able to differentiate into myofibroblasts and then affect tumor stroma remodeling, but the mechanism is not clear. This study was aimed to ascertain whether TGF $31-S m a d$ pathway was involved in this process. In order to verify the hypothesis, we designed an in vitro experiment and an animal experiment. The in vitro experiment aimed to find out whether TGF 1 1-Smad pathway was involved in the VX2 culture supernatant-induced differentiation of MSCs to myofibroblasts. The animal experiment aimed to discover whether TGF $\beta 1-S m a d$ pathway was involved in the process that MSCs could affect tumor stroma remodeling.

\section{Animals}

Fifty male New Zealand white rabbits (3 months old, weight $1.5-2.0 \mathrm{~kg}$ ) were purchased from Shandong Academy of Agricultural Sciences. The animal protocols were approved by the Institutional Animal
Care and Use Committee of Qilu Hospital, Shandong University.

\section{The groups of in vitro experiment}

There were five groups for the in vitro experiment, named as control A, control B, control C, test $A$, test $B$, respectively. For group control A, F2 passage MSCs were cultured in DMEM-LG with 10\% calf-serum, 30\% VX2 culture supernatant. For group control B, F2 passage MSCs, which had been transfected by blank liposomes, were cultured in DMEM-LG with $10 \%$ calf-serum, $30 \%$ VX2 culture supernatant. For group control C, F2 passage MSCs were cultured in DMEM-LG with $10 \%$ calf-serum. For group test A, F2 passage MSCs, which had been transfected with siRNA targeting TGF $\beta 1$ receptor by liposomes, were cultured in DMEM-LG with $10 \%$ calf-serum, 30\% VX2 culture supernatant. For group test B, F2 passage MSCs, which had been transfected with siRNA targeting Smad2 by liposomes, were cultured in DMEM-LG with $10 \%$ calf-serum, 30\% VX2 culture supernatant. The expression of a-SMA and Vimentin in MSCs was detected by westernblot 14 days later. 10 samples of each group, $10^{7}$ MSCs in each sample, were tested.

\section{The groups of animal experiment}

Fifty male New Zealand white rabbits were randomly divided into control 1, control 2, control 3, test 1 , test 2 , with ten rabbits in each group. For group control 1 , the cell suspension, containing $10^{6}$ autologous MSCs and $10^{6}$ VX2, was injected into the bladder submucosa. For group control 2, the cell suspension, containing $10^{6}$ autologous MSCs transfected by blank liposomes and $10^{6} \mathrm{VX} 2$, was injected into the bladder submucosa. For group control 3, the cell suspension, containing $10^{6} \mathrm{VX} 2$, was injected into the bladder submucosa. For group test 1 , the cell suspension, containing $10^{6}$ autologous MSCs transfected with siRNA targeting TGF $\beta 1$ receptor by liposomes and $10^{6} \mathrm{VX} 2$, was injected into the bladder submucosa. For group test 2, the cell suspension, containing $10^{6}$ autologous MSCs transfected with siRNA targeting Smad2 by liposomes and $10^{6} \mathrm{VX} 2$, was injected into the bladder submucosa.

The animals were sacrificed 4 weeks later. The expression of TGF $\beta 1$, EGF, FAPa, MMP9 were detected by westernblot, and the tumor size was recorded.

\section{MSCs isolation, cultivation and identification}

The process of MSCs isolation, cultivation and identification was the same as we previously described ${ }^{[12]}$. MSCs with CD34(-), CD44(+) and CD45(-) were used for study. 


\section{VX2 tumor cells isolation and cultivation}

The VX2 tumor carrier rabbit was presented by Radiology Department of Qilu Hospital. The process of VX2 tumor cells isolation and cultivation was the same as we previously described [12].

\section{Tumor inoculation procedure}

We established tumor model by injecting mixed cell suspensions under the bladder mucosa. After the exposure of the urinary bladder through the lower abdominal incision under sterile conditions, we made a small incision $(0.8 \mathrm{~cm})$ in the bladder wall, and perpendicularly inoculated the cell suspension into the bladder wall. Then bladder wall and abdominal wall were stitched in turn[12].(Figure 1)

\section{Cell transfection}

According to LipofectamineTM 2000 liposome transfection reagent instruction (Lipofect2000, Invitrogen), F2 passage MSCs were digested by pancreatic enzyme, and then were counted. They were inoculated at $2 \times 10^{5} / \mathrm{mL}$. When fusion of cell growth was $70 \%-80 \%$, OD SiRNA diluted by OptiMEM ${ }^{\circledR}$ and LipofectamineTM 2000 diluted by OptiMEM ${ }^{\circledR}$ Medium were mixed, and incubated for 20 min; Mixture was added to each hole, and incubated at $37{ }^{\circ} \mathrm{C}$ and $5 \% \mathrm{CO} 2$, saturated humidity. After $9 \mathrm{hr}$ they were cultured by DMEM containing $10 \%$ FBS/F-12 media, and then were screened and cultured.

\section{Western blotting}

Briefly, the protein concentrations of all samples were measured by BCA protein assay kit (Pierce, USA) after protein extraction. After boiled for $5 \mathrm{~min}$, the protein samples were fractionated by SDS-PAGE (10-15\% polyacrylamide gels) and transferred to PVDF membrane (Millipore, Bedford, MA, USA). The samples were blocked with milk powder for $1 \mathrm{hr}$ at room temperature and then incubated with primary antibodies (Abcam China) as well as calcineurin and NFATc3 (Santa Cruz Biotechnology Inc., Santa Cruz, CA USA) at $4^{\circ} \mathrm{C}$ overnight. After washing, the membranes were incubated with a secondary antibody (Santa Cruz Biotechnology Inc., Santa Cruz, CA USA) for $1 \mathrm{hr}$ at room temperature. Western blot bands were quantified using Gel analysis system by measuring the integrated optical density (IOD). The protein expression intensity was quantified by relative optical density (ROD). The ROD was defined as protein IOD/actin IOD.

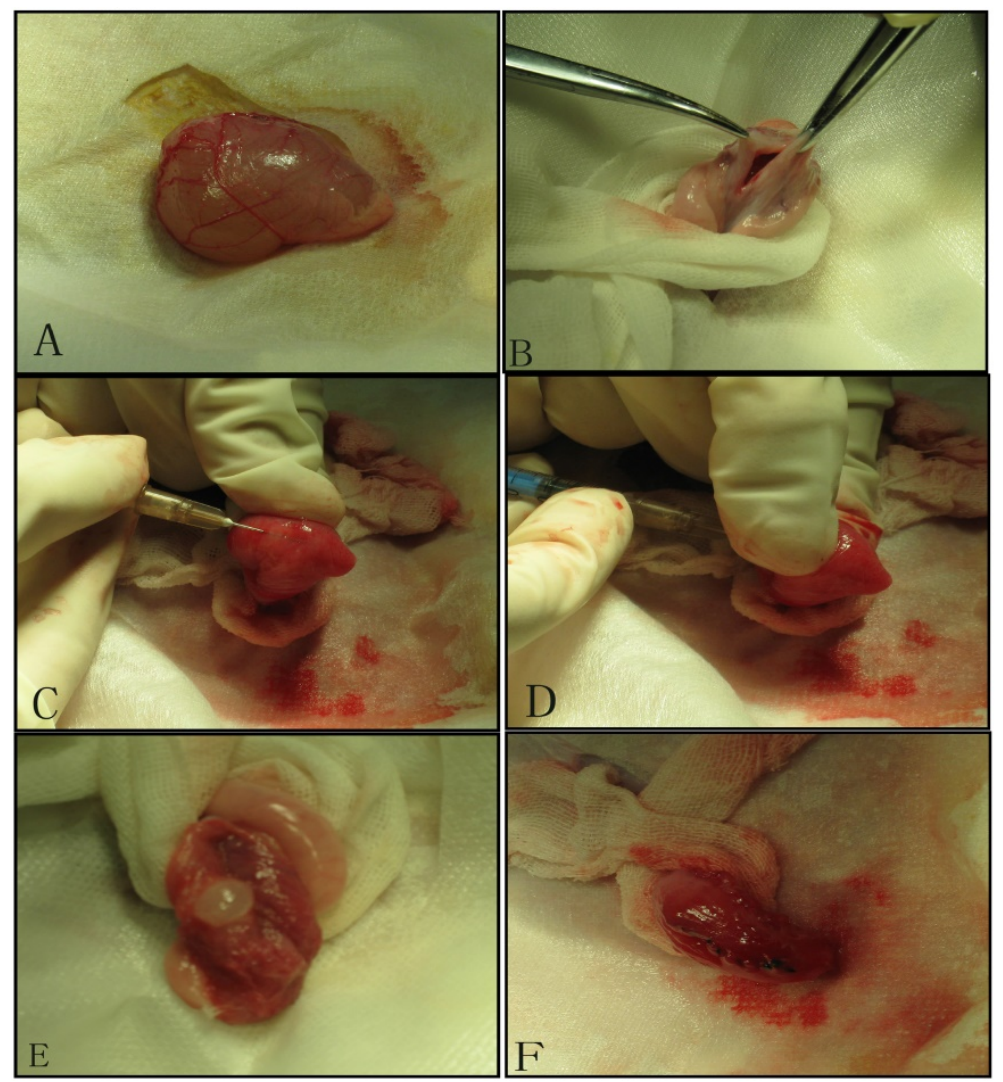

Figure 1. Tumor inoculation procedure: A. Exposure the bladder; B. Cystotomy along the midline; C. 1 ml needle (containing 300 ul of cell suspension) punctures into the mucosa on the side wall of the bladder; D. After about $1.5 \mathrm{~cm}$ of immersion in the submucosa of the bladder, the thumb of the left hand presses against point of the needle, and $300 u$ of cell suspension is injected under the mucosa; E. Blisters formed by cell suspension after injection; F. Close the bladder with absorbable suture. 


\section{Statistical analysis}

All values were expressed as mean \pm standard deviation $(\bar{x} \pm S)$. SPSS 17.0 was used to deal with the data, and $t$ test was used to determine statistical differences between two groups. $\mathrm{P}<0.05$ was considered as significant difference.

\section{Results}

The in vitro experiment showed that blocking the expression of TGF $\beta 1$ receptor or Smad2 affected the differentiation of MSCs to myofibroblast

The ROD values of a-SMA and Vimentin were $0.212 \pm 0.018$ and $0.289 \pm 0.036$ when MSCs were cultured with DMEM-LG in group control C. When MSCs were cultured with DMEM-LG and 30\% VX2 culture supernatant in group control $A$, the ROD values of a-SMA and Vimentin were both significantly increased $(0.641 \pm 0.026,0.476 \pm 0.029)$, which indicated VX2 culture supernatant could induce the differentiation of MSCs to myofibroblasts. However, compared with group control A, the ROD values of a-SMA and Vimentin were both significantly decreased when the expression of TGF $\beta 1$ receptor was blocked by siRNA in group test A $(0.302 \pm 0.021,0.378 \pm 0.040)$. Besides, both the ROD values of a-SMA and Vimentin were significantly decreased when the expression of Smad 2 was blocked by siRNA in group test B $(0.270 \pm 0.021,0.368 \pm 0.048)$. The results indicated that both TGF $\beta 1$ receptor and Smad2 were involved in the differentiation of MSCs to myofibroblasts.(Figure 2)

\section{TGF $\beta 1$ receptor and Smad2 participate in the MSCs-induced acceleration of VX2 tumor growth}

All rabbits were sacrificed 4 weeks after tumor inoculation. The maximum diameter was recorded. The mean maximum diameter of group control 3 was $2.01 \pm 0.28 \mathrm{~cm}$. When VX2 cells and MSCs were co-inoculated in group control 1, the mean maximum diameter was significantly increased $(2.87 \pm 0.43 \mathrm{~cm})$, which indicated that MSCs were conducive to VX2 tumor growth. In group test 1 , the expression of TGF $\beta 1$ receptor of MSCs was blocked, then these cells were co-inoculated with VX2 cells. Compared with group control 1, the mean maximum diameter was significantly decreased $(2.38 \pm 0.36 \mathrm{~cm})$. The same tendency was also found in group test 2. After the expression of Smad2 of MSCs was blocked, these MSCs were co-inoculated with VX2 cells. Compared with group control 1, the mean maximum diameter was also significantly decreased $(2.28 \pm 0.32 \mathrm{~cm})$. (Figure 3)
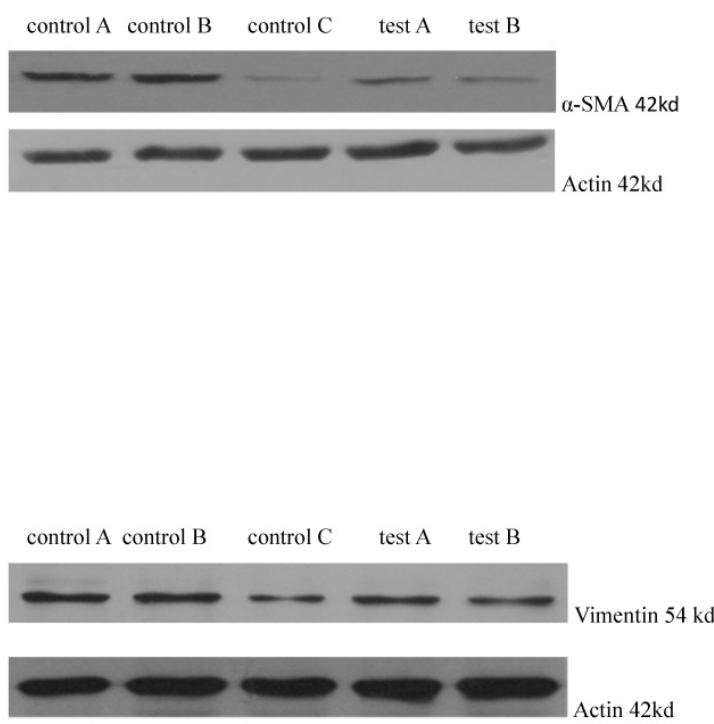

The ROD values of $\alpha$-SMA

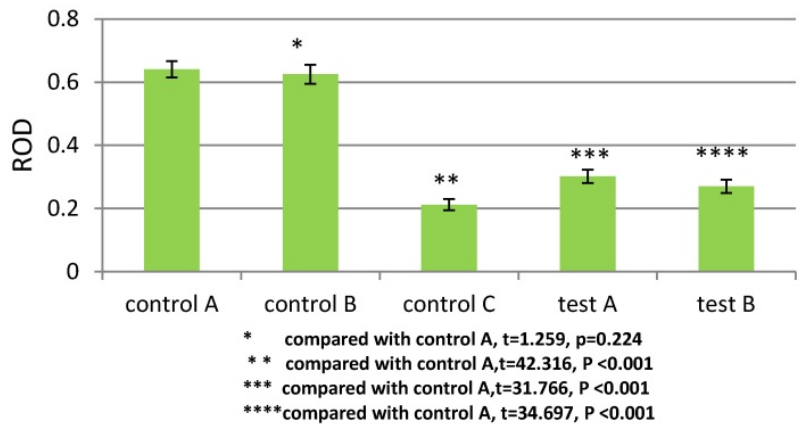

The ROD values of Vimentin

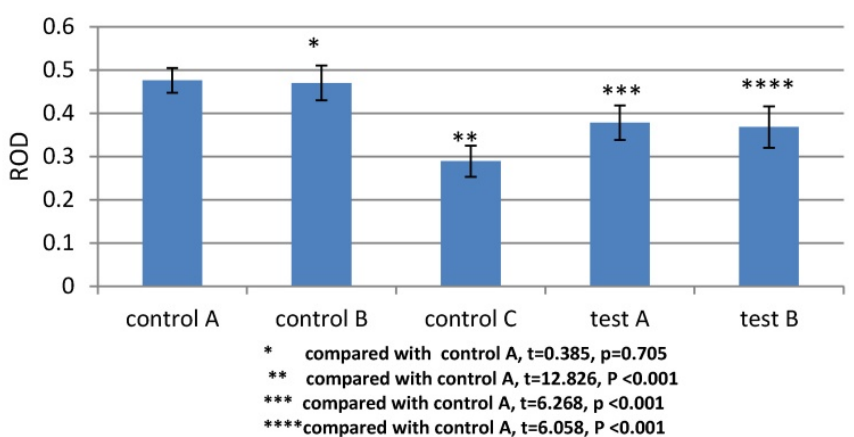

Figure 2. The expression of $\alpha-S M A$ and Vimentin in MSCs was detected by westernblot. Control B was used to test whether or not liposome could affect the result. Compared with control $A, p>0.05$ indicated liposome did not affect the result. The comparison between group control $C$ and group control $A$ indicated that $V X 2$ culture supernatant could induce the expression of $\alpha-S M A$ and Vimentin. Blockage of the expression of TGF $\beta 1$ receptor (group test A) or Smad2 (group test B) would both lead to decreased expression of $\alpha$-SMA and Vimentin. 


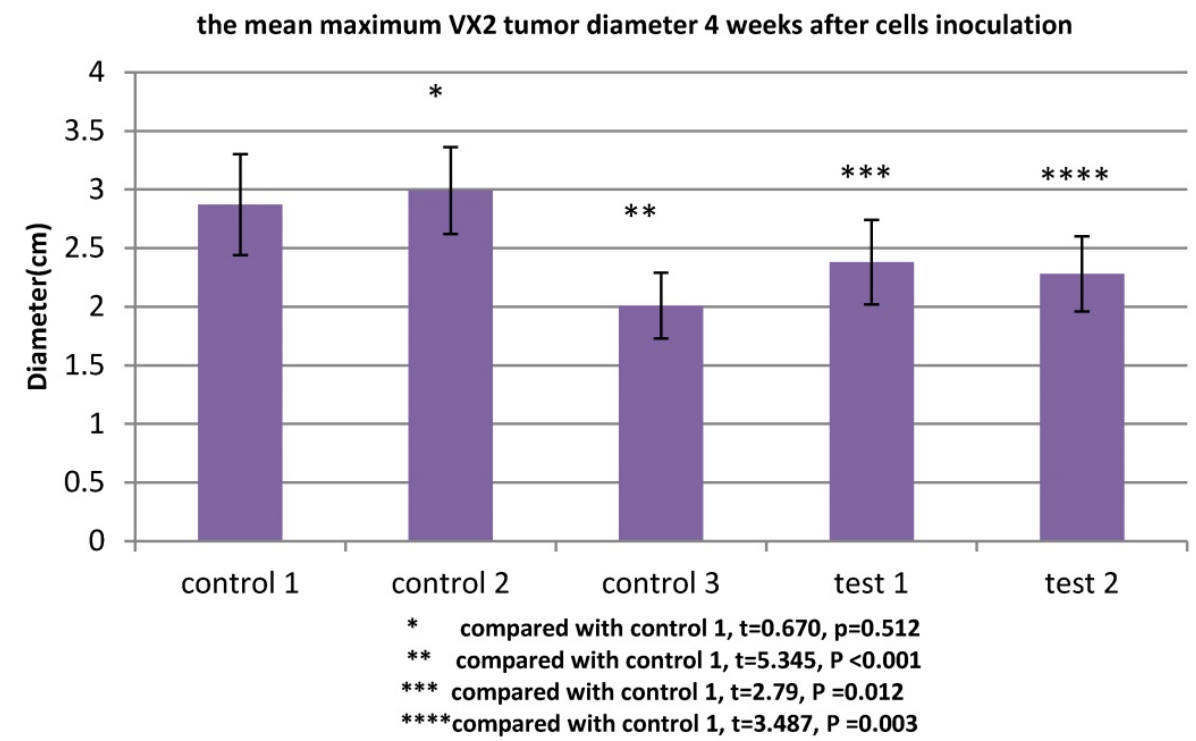

Figure 3. The maximum diameter of $\mathrm{VX} 2$ bladder tumor 4 weeks after tumor inoculation. Control 2 was used to test whether or not liposome could affect the result. Compared with control 1, p>0.05 indicated liposome did not affect the result. The comparison between group control 3 and group control 1 indicated that MSCs were conducive to VX2 tumor growth. The expression of TGF 31 receptor and Smad2 of MSCs were blocked in group test 1 and group test 2, respectively. Compared with group control 1, the mean maximum tumor diameter was significantly decreased in group test 1 and group test 2

\section{TGF $\beta 1$ receptor and Smad 2 mediated the facilitation of MSCs to the expression of TGF $\beta 1$ and EGF in VX2 tumor tissue}

The ROD values of TGF $\beta 1$ and EGF were $0.264 \pm 0.054$ and $0.459 \pm 0.090$ in group control 3 . When VX2 cells and MSCs were co-inoculated in group control 1, the ROD values of TGF $\beta 1$ and EGF were both significantly increased $(0.899 \pm 0.124,1.053 \pm 0.107)$, which indicated that MSCs facilitated the expression of these two growth factors in VX2 tumor tissue. Compared with group control 1, the ROD values of TGF $\beta 1$ and EGF were both significantly decreased $(0.333 \pm 0.074,0.714 \pm 0.072)$ when the expression of TGF $\beta 1$ receptor of MSCs was blocked in group test 1 . Moreover, both the ROD values of TGF $\beta 1$ and EGF were significantly decreased when the expression of Smad2 of MSCs was blocked in group test 2 $(0.346 \pm 0.089,0.723 \pm 0.084)$. The results indicated that both TGF $\beta 1$ receptor and Smad 2 were involved in the process that MSCs facilitated the expression of TGF $\beta 1$ and EGF in VX2 tumor tissue. (Figure 4)

\section{TGF $\beta 1$ receptor and Smad 2 mediated the facilitation of MSCs to the expression of FAPa and MMP9 in VX2 tumor tissue}

The ROD values of FAPa and MMP9 were $0.072 \pm 0.036$ and $1.012 \pm 0.087$ in group control 3. When VX2 cells and MSCs were co-inoculated in group control 1, the ROD values of FAPa and MMP9 were both significantly increased $(0.222 \pm 0.041,1.147 \pm 0.121)$. When the expression of TGF $\beta 1$ receptor of MSCs was blocked in group test 1 , the ROD values of FAPa and MMP9 were both significantly decreased $(0.149 \pm 0.046$,
$1.036 \pm 0.108)$ compared with group control 1. And both The ROD values of FAPa and MMP9 were significantly decreased when the expression of Smad2 of MSCs was blocked in group test $2(0.153 \pm 0.056$, $1.022 \pm 0.120)$. The results indicated that both TGF $\beta 1$ receptor and Smad2 were involved in the process that MSCs facilitated the expression of FAPa and MMP9 in VX2 tumor tissue. (Figure 5)

\section{Discussion}

Activation of microenvironment is a critical step for tumor growth and development ${ }^{[14-16]}$. Activated mesenchymal cells produce a large amount of extracellular matrix components, growth factors, and matrix remodeling proteins, thereby forming a microenvironment that is conducive to tumor growth and proliferation. These mesenchymal cells mainly include fibroblasts, myofibroblasts, endothelial cells, and some immune cells ${ }^{[17]}$. Although all of the above cells play a role in the growth and development of tumors, it is currently believed that myofibroblasts are especially important for tumor growth, invasion and metastasis ${ }^{[18-20]}$. In the activated interstitial microenvironment, myofibroblasts produce some proteases such as fibroblast activation protein, metalloproteinases, urokinase, plasminogen activator. In addition, they also synthesize some extracellular matrix components such as collagen I, collagen III, fibronectin, mucin, polysaccharide proteins. Some reports suggest that myofibroblasts can secrete certain growth factors to promote the development of cancer, such as TGF $\beta 1$, EGF, platelet-derived growth factor, fibroblast growth factor, hepatocyte growth factor, keratinocyte growth factor, stem cell factor ${ }^{21,22]}$. 
Control $1 \quad$ control 2 control $3 \quad$ test $1 \quad$ test 2

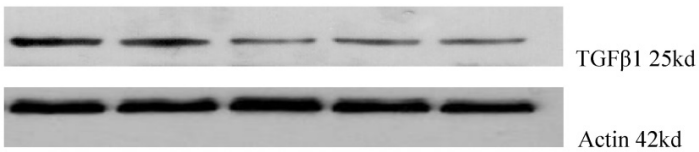

control

control 2 control 3 test 1

test 2

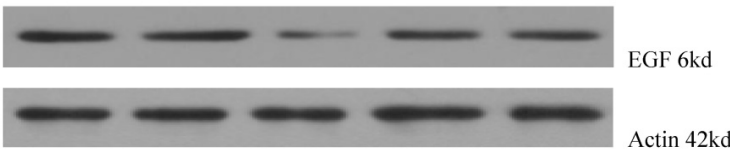

The ROD values of TGF $\beta 1$

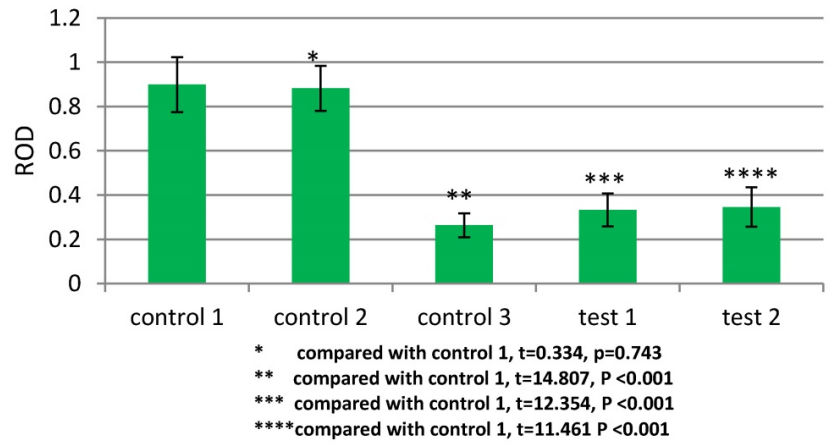

The ROD values of EGF

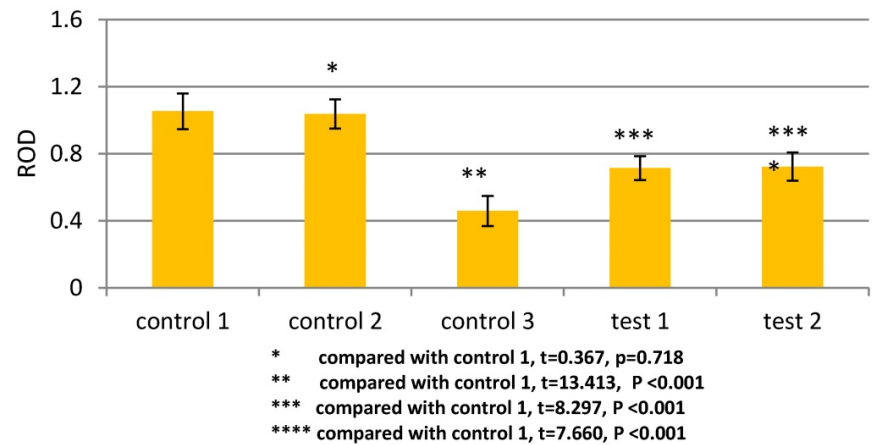

Figure 4. The expression of TGF $\beta 1$ and EGF in vx2 tumor tissue was detected by westernblot. Control 2 was used to test whether or not liposome could affect the result. Compared with control 1, $>>0.05$ indicated liposome did not affect the result. The comparison between group control 3 and group control 1 indicated that MSCs could enhance the expression of TGF $\beta 1$ and EGF. Blockage of the expression of TGF $\beta 1$ receptor (group test 1) or Smad2 (group test 2) would both lead to decreased expression of TGF $\beta 1$ and EGF.
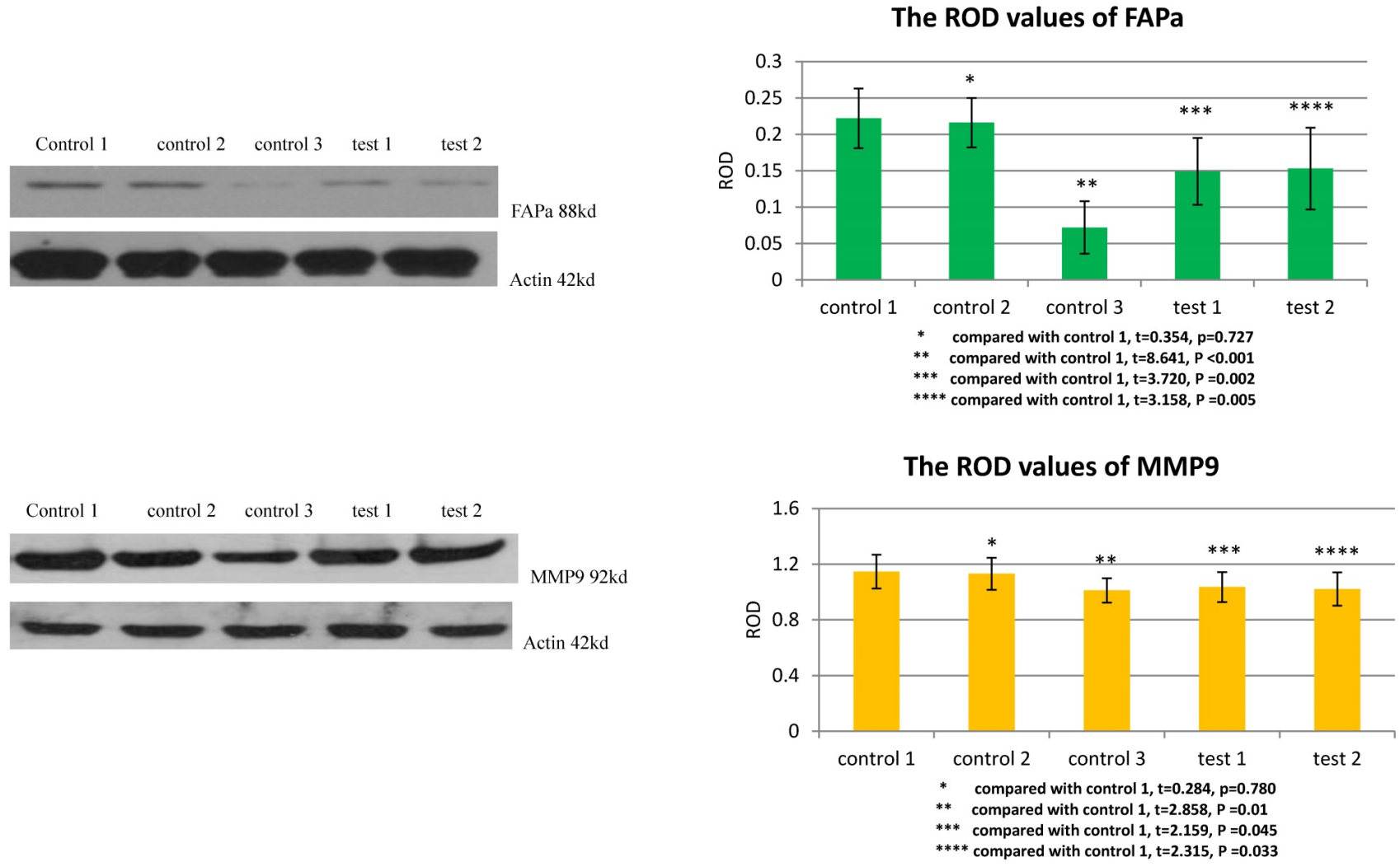

Figure 5. The expression of FAPa and MMP9 in vx2 tumor tissue was detected by westernblot. Control 2 was used to test whether or not liposome could affect the result. Compared with control 1, p $>0.05$ indicated liposome did not affect the result. The comparison between group control 3 and group control 1 indicated that MSCs could enhance the expression of FAPa and MMP9. Blockage of the expression of TGF 31 receptor (group test 1) or Smad2 (group test 2) would both lead to decreased expression of FAPa and MMP9. 
In the tumor stroma, myofibroblasts are mainly derived from fibroblasts. Since many scholars believe that the fibroblasts in the granulation tissue can be derived from some progenitor cells in the circulation [23-25], Alexis et al therefore believe that the circulating cells will also migrate to tumor tissue and differentiate into myofibroblasts when the tumor grows to a certain size and local fibroblasts are not sufficient[26]. Ishii's findings further indicate that myofibroblasts in tumor stroma are derived from bone marrow, and that the higher the tumor stage, the greater the number of myofibroblasts derived from the bone marrow ${ }^{[2]}$. However, this study only showed that myofibroblasts in tumor stroma could also derive from the bone marrow but did not specify which cells they derived from. Jeon's findings indicate that lysophosphatidic acid secreted by tumors can induce the differentiation of MSCs into myofibroblast-like cells[13]. Mishra found that the expression of a-SMA, a marker of myofibroblast, was significantly increased in bone marrow mesenchymal cells induced by tumor-conditioned medium, and the induced MSCs significantly promoted tumor growth both in vitro and in vivo[28]. Therefore, at least part of myofibroblasts in tumor tissue should derive from bone marrow MSCs. In addition to bone marrow, MSCs are widely distributed in other parts of the body. Therefore, myofibroblasts in tumors may also derive from MSCs in other sites. In addition, Bagley's results indicate that MSCs are mainly distributed in tumor stroma after entering tumor tissue, and they mainly differentiate into myofibroblast precursor cells, fibroblasts ${ }^{[29]}$. In view of the important role of myofibroblasts in tumor growth, invasion, and metastasis, one of the mechanisms by which MSCs facilitate tumor growth is to promote tumor growth by differentiated myofibroblasts.

Smad2/Smad3 is the first signaling molecule in TGF $\beta 1$ - Smad signaling pathway. As a direct substrate of the TGF $\beta$ superfamily, Smad2/Smad3 plays a key mediating role in the transmission of TGF $\beta 1$ signaling from cytoplasm to nucleus ${ }^{[30,31]}$. In the previous studies, we found that MSCs could differentiate into myofibroblasts under the induction of tumor microenvironment, and further promote the growth and development of tumors by promoting remodeling of tumor interstitial microenvironment ${ }^{10}$, 12]. In this study, we also found that MSCs could differentiate into myofibroblasts under the induction of tumor cell culture supernatant. Blocking the expression of TGF $\beta 1$ receptor or Smad2 in MSCs can significantly down-regulate the expression of a-SMA and Vimentin. This indicates that TGF $\beta 1-S m a d$ signaling pathway is involved in the differentiation of MSCs into myofibroblasts. We further investigated whether TGF 31 -Smad signaling pathway was involved in the process that MSCs facilitated tumor growth and tumor interstitial microenvironment remodeling. We found that blocking the expression of TGF $\beta 1$ receptor or Smad2 in MSCs could down-regulate the tumor growth promoting effect of MSCs, and significantly down-regulate the expression of TGF $\beta 1$, EGF, FAPa and MMP9, which were closely related to the activation and remodeling of tumor interstitial microenvironment. We speculate that one of the reasons for this result is that blocking TGF $\beta 1$-Smad signaling pathway affects the differentiation of MSCs into myofibroblasts.

Although most studies indicate that MSCs facilitate tumor growth and metastasis, some studies indicate that MSCs can inhibit tumor development. However, the vast majority of these studies are the results of in vitro cell experiments or immunodeficient mice, and the MSCs used are allogeneic. So one merit of this study is that the animals used in this study are normal rabbits and MSCs used in every rabbit are autologous, which avoids the immune interference on the study results.

\section{Conclusions}

Under the induction of tumor microenvironment, MSCs can differentiate into myofibroblasts and then affect tumor interstitial microenvironment remodeling. This process is mediated by TGF $\beta 1-S m a d 2$ pathway.

\section{Abbreviations}

MSCs: Mesenchymal stem cells; TGF $\beta 1$ : transforming growth factor $\beta 1$; EGF: epidermal growth factor; FAPa: fibroblast activation protein alpha; MMP9: matrix metalloprotein 9; PBS: phosphate buffered saline; DMEM-LG: dulbecco's modified eagle medium-low glucose; IOD: intergrated optical density; ROD: relative optical density.

\section{Acknowledgements}

This work was supported by National Natural Science Foundation of China grant (no.30900549) (the design of the study and collection, analysis, and interpretation of data and in writing the manuscript), Natural Science Foundation of Shandong Province grant (no. ZR2009CQ026) (the design of the study and collection, analysis, and interpretation of data and in writing the manuscript), Shandong provincial key research and development plan (no. 2017GSF218008) (collection, analysis, and interpretation of data), and Scientific research start fund of Qilu Hospital (Qingdao) of Shandong University (no. QDKY2016ZD04) (in writing the manuscript). 


\section{Authors' contributions}

JC designed and coordinated the study. QY analyzed data and wrote the paper. YZ did animal and in vitro experiments, flow cytometry work; ZX contributed experimental design and data analysis. All authors read and approved the final manuscript.

\section{Competing Interests}

The authors have declared that no competing interest exists.

\section{References}

1. Sordi $\mathrm{V}$, Malosio ML, Marchesi F, et al. Bone marrow mesenchymal stem cells express a restricted set of functionally active chemokine receptors capable of promoting migration to pancreatic islets. Blood. 2005;106:419-27.

2. Sordi V. Mesenchymal stem cell homing capacity. Transplantation. 2009; 87 (Suppl 9):S42-S45.

3. Sato Y, Araki H, Kato J, et al. Human mesenchymal stem cells xenografted directly to rat liver are differentiated into human hepatocytes without fusion. Blood. 2005; 106:756-63.

4. Yamaguchi Y, Kubo T, Murakami T, et al. Bone marrow cells differentiate into wound myofibroblasts and accelerate the healing of wounds with exposed bones when combined with an occlusive dressing. $\mathrm{Br} J$ Dermatol. 2005;152:616-22.

5. Ridge SM, Sullivan FJ, Glynn SA. Mesenchymal stem cells: key players in cancer progression. Mol Cancer. 2017;16:31.

6. Studeny M, Marini FC, Dembinski JL, et al. Mesenchymal stem cells: potential precursors for tumor stroma and targeted-delivery vehicles for anticancer agents. J Natl Cancer Inst. 2004; 96:1593-603.

7. Coffelt SB, Marini FC, Watson K, et al. The pro-inflammatory peptide LL-37 promotes ovarian tumor progression through recruitment of multipotent mesenchymal stromal cells. Proc Natl Acad Sci U S A. 2009; 106:3806-11.

8. Spaeth E, Klopp A, Dembinski J, et al. Inflammation and tumor microenvironments: defining the migratory itinerary of mesenchymal stem cells. Gene Ther. 2008; 15:730-8

9. Nakamizo A, Marini F, Amano T, et al. Human bone marrow-derived mesenchymal stem cells in the treatment of gliomas. Cancer Res. 2005; 65:3307-18.

10. Chen J, Xu ZS, Zhao HF, et al. Distribution and differentiation of marrow mesenchymal cells in tumor tissue: experimental with rabbits. Zhonghua Yi Xue Za Zhi. 2007; 87:2361-4.

11. Karnoub AE, Dash AB, Vo AP, et al. Mesenchymal stem cells within tumor stroma promote breast cancer metastasis. Nature. 2007; 449:557-63.

12. Chen J, Ma L, Zhang N, et al. Mesenchymal Stem Cells Promote Tumor Progression via Inducing Stroma Remodeling on Rabbit VX2 Bladder Tumor Model. Int J Biol Sci. 2018; 14:1012-21.

13. Jeon ES, Moon HJ, Lee MJ, et al. Cancer-derived lysophosphatidic acid stimulates differentiation of human mesenchymal stem cells to myofibroblast-like Cells. Stem Cells. 2008; 26:789-97.

14. Condon MS. The role of the stromal microenvironment in prostate cancer. Semin Cancer Biol. 2005;15:132-7.

15. Bhowmick NA, Neilson EG, Moses HL. Stromal fibroblasts in cancer initiation and progression. Nature. 2004; 432: 332-7.

16. McCuaig $\mathrm{R}, \mathrm{Wu} \mathrm{F}$, Dunn J, et al. The biological and clinical significance of stromal-epithelial interactions in breast cancer. Pathology. 2017; 49:133-40

17. Mahale J, Smagurauskaite G, Brown K, et al. The role of stromal fibroblasts in lung carcinogenesis: A target for chemoprevention? Int J Cancer. 2016;138:30-44.

18. Powell DW, Adegboyega PA, Di Mari JF, et al. Epithelial cells and their neighbors I. Role of intestinal myofibroblasts in development, repair, and cancer. Am J Physiol Gastrointest Liver Physiol. 2005; 289:G2-7.

19. Galiè M, Sorrentino $C$, Montani M, et al. Mammary carcinoma provides highly tumourigenic and invasive reactive stromal cells. Carcinogenesis. 2005; 26:1868-78.

20. Rodrigues PC, DA Costa Miguel MC, DE Aquino SN, et al. Stromal myofibroblasts in potentially malignant and malignant lesions of the oral cavity. Oncol Lett. 2015; 9:667-70.

21. Frazier KS, Grotendorst GR. Expression of connective tissue growth factor mRNA in the fibrous stroma of mammary tumors. Int J Biochem Cell Biol. 1997; 29:153-61.

22. Shimo $T$, Nakanishi $T$, Nishida $T$, et al. Connective tissue growth factor induces the proliferation, migration, and tube formation of vascular endothelial cells in vitro, and angiogenesis in vivo. J Biochem. 1999; 126:137-45.

23. Abe R, Donnelly SC, Peng T, et al. Peripheral blood fibrocytes: differentiation pathway and migration to wound sites. J Immunol. 2001; 166:7556-62.

24. Kalka C, Masuda H, Takahashi T, et al. Vascular endothelial growth factor(165) gene transfer augments circulating endothelial progenitor cells in human subjects. Circ Res. 2000; 86:1198-202.
25. Yang L, Scott PG, Giuffre J, et al. Peripheral blood fibrocytes from burn patients: identification and quantification of fibrocytes in adherent cells cultured from peripheral blood mononuclear cells. Lab Invest. 2002; 82: 1183-92.

26. Alexis D, Christelle G, Giulio G. The stroma reaction myofibroblast: a key player in the control of tumor cell behavior. Int J Dev Biol. 2004; 48:509-17.

27. Ishii $G$, Sangai $T$, Oda $T$, et al. Bone-marrow-derived myofibroblasts contribute to the cancer-induced stromal reaction. Biochem Biophys Res Commun. 2003; 309:232-40.

28. Mishra PJ, Mishra PJ, Humeniuk R, et al. Carcinoma-associated fibroblast-like differentiation of human mesenchymal stem cells. Cancer Res. 2008; 68:4331-9.

29. Bagley RG, Weber W, Rouleau C, et al. Human mesenchymal stem cells from bone marrow express tumor endothelial and stromal markers. Int J Oncol. 2009; 34:619-27.

30. Schiffer M, von Gersdorff G, Bitzer M, et al. Smad proteins and transforming growth factor-beta signaling. Kidney Int Suppl. 2000; 77:S45-S52.

31. ten Dijke P, Hill CS. New insights into TGF-beta-Smad signaling. Trends Biochem Sci. 2004; 29: 265-73. 